\title{
Time Disturbances and Filtering of Sensors Signals in Tolerant Multi-product Job-shops with Time Constraints
}

\author{
Nabil Jerbi, Simon Collart Dutilleul, Etienne Craye, Mohamed Benrejeb
}

\begin{abstract}
This paper deals with supervision in critical time manufacturing jobshops without assembling tasks. Such systems have a robustness property to deal with time disturbances. A filtering mechanism of sensors signals integrating the robustness values is proposed. It provides the avoidance of control freezing if the time disturbance is in the robustness intervals. This constitutes an enhancement of the filtering mechanism since it makes it possible to continue the production in a degraded mode providing the guarantees of quality and safety. When a symptom of abnormal functioning is claimed by the filtering mechanism, it is imperative to localize the time disturbance occurrence. Based upon controlled P-time Petri nets as a modeling tool, a series of lemmas are quoted in order to build a theory dealing with the localization problem.
\end{abstract}

Keywords: P-time Petri net, sensor signal, filtering, time disturbance, localization

\section{Introduction}

This paper concerns critical time manufacturing job-shops. For each operation is associated a time interval. Its lower bound indicates the minimum time needed to execute the operation. The non respect of this value means that the operation was not achieved. The upper bound fixes the maximum time to not exceed otherwise the quality of the product is deteriorated. Such systems have a robustness property in order to maintain product quality when there are time disturbances [1], [2]. The robustness is defined as the ability of the system to preserve the specifications facing some expected or unexpected variations. So the robustness characterizes the capacity to deal with disturbances. The robustness is interpreted into different specializations. The passive robustness is based upon variations included in validity time intervals. There is no control loop modification to preserve the required specifications. On the other hand, active robustness uses observed time disturbances to modify the control loop in order to satisfy these specifications. Therefore, the robustness intervals must be integrated in the filtering mechanism of sensors signals. Furthermore, the observability of time disturbances occurrence is a fundamental data necessary for the control loop modification. It is also an important aspect of the maintaining task [3], [4]. When an abnormal functioning is claimed, it is important to know the initial occurrence of the disturbance. The localization problem is really difficult in robust systems since the rejection of disturbances may hide them [5].

The first part of this paper presents a filtering mechanism of sensors signals taking into account the robustness values. The second part considers the localization of time disturbances. It is necessary to perform this task when the disturbance value passes through the filter. Controlled P-time Petri nets are used for modeling the considered workshops. Afterward, the localization problem of time disturbances in critical time manufacturing systems is tackled. Some definitions and lemmas are quoted in order to build a theory dealing with such problems.

\section{Robustness integration in the filtering of sensors signals}

At the occurrence of a dysfunction in a manufacturing workshop, it is crucial to react as soon as possible to maintain the productivity and to ensure the safety of the system. It has been recognized that 
the real time piloting, without human intervention, has a significant contribution regarding this type of problem [6], [7].

In the category of the workshops concerned by this article, the operations have temporal constraints which must be imperatively respected. The violation of these constraints can blame the health of the consumers. Thus, the detection of a constraint violation must automatically cause the stop of the production. On the other hand, when taking into account the system robustness, it is proven that this type of violation did not take place. In this case, we plan to maintain the production while describing it as degraded production [8]. Of course, the product is not degraded, but the production is degraded because the deliveries moments of the products are not those envisaged initially. It is this context which we propose to integrate in the generation of symptoms as it was presented in [9].

The finality of this section is not to contribute to the state of the art of the monitoring-diagnosis, but to show how the knowledge of the robustness could make the supervision more efficient. By considering this criterion, the filtering of sensors signals of [9] appears pedagogically interesting.

\subsection{Symptoms generation}

The idea consists in modeling any operation from a temporal approach. At each operation $A_{i}$ is associated a sensor signal $C R_{i}$. At each sensor signal $C R_{i}$ is associated a temporal window $\left[\Delta t_{m / C R_{i}}, \Delta t_{M / C R_{i}}\right]$ (Figure 1). $C R_{i}$ is valid only inside this window. $\Delta t_{m / C R_{i}}$ and $\Delta t_{M / C R_{i}}$ are defined relatively to the beginning of the operation $A_{i}$ (Start-Event). The filtering principle is to position the temporal window of each sensor signal $C R_{i}$ when his Start-Vent was received. Two types of symptoms are distinguished [9].

Symptoms type I noted $S_{i}^{1}$ : this class of symptoms corresponds to awaited sensor signal which is not received at $\Delta t_{M / C R_{i}}$. The detection mechanism of this symptom type corresponds to the traditional mechanism of watchdog, but implemented in a separate way of the control. Symptoms type II noted $S_{i}^{2}$ : it is generated by the occurrence of a sensor signal which is not expected. Two cases are considered. The first one corresponds to an action but its sensor signal occurs before the validation interval. The second case corresponds to the occurrence of a sensor signal in absence of any order which can create it.

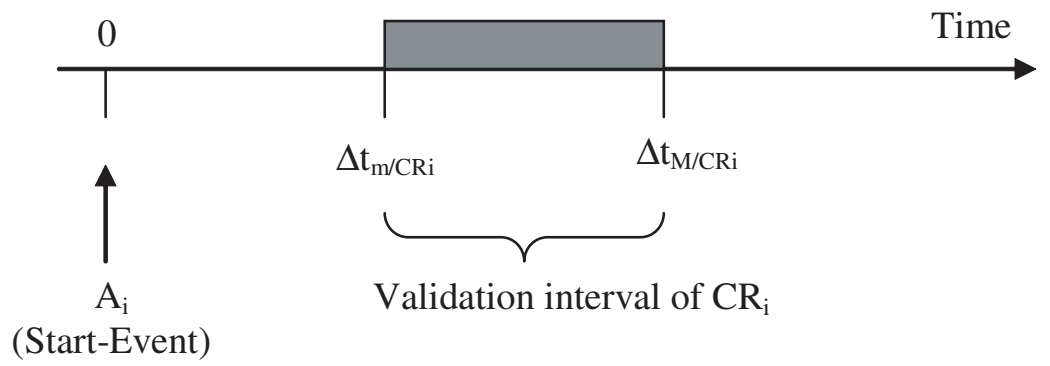

Figure 1: Operation associated model [9]

\subsection{Robustness integration}

The tool used to represent the filtering mechanism is the interpreted T-time Petri net. Initially, we point out the definition of the interpreted T-time Petri net. After, we give the filtering mechanism of sensors signals integrating the two properties of passive and active robustness.

Definition 1. A T-time Petri net is given by a pair $\left\langle R ; I S^{\prime}\right\rangle$, where $R$ is a Petri net and $I S^{\prime}: T \rightarrow$ $\left(Q^{+}\right) \times\left(Q^{+} \cup+\infty\right)[10]$. 
Definition 2. Interpreted T-time Petri net is a T-time Petri net including an operative part whose state is defined by a set of variables. This state is modified by the operations associated to the places. It determines the value of the conditions (predicates) which are associated to the transitions.

The mechanism of watchdog is easily represented by an interpreted T-time Petri net. For example, figure 2 shows the detection of a normal state and an abnormal one. If the sensor signal arrives in $\left[t_{m}, t_{M}[\right.$, the system is in a normal state. If the sensor signal arrives at the instant $t_{M}$, the system is in an abnormal one.

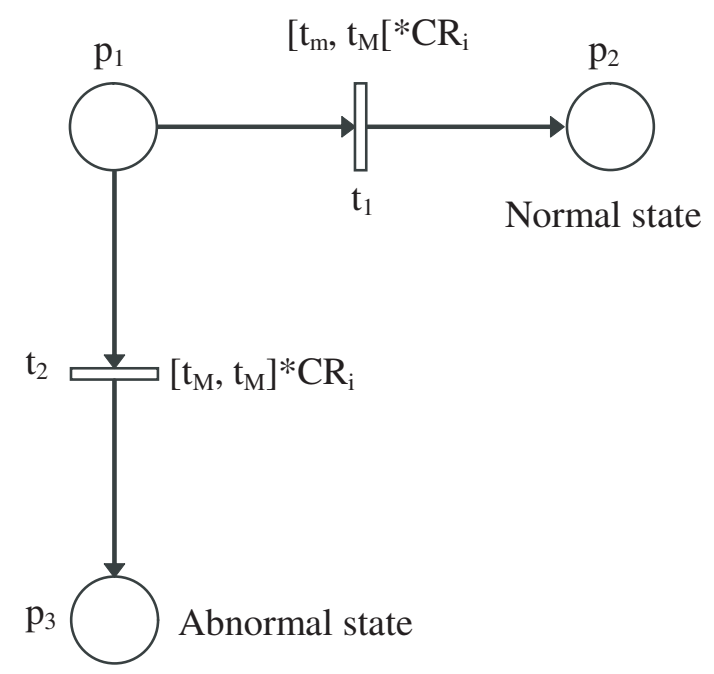

Figure 2: Watchdog mechanism with interpreted T-time Petri net

Within the framework of the robustness integration in the supervision of manufacturing systems with time constraints, we define, figure 3, five time intervals namely:

$I_{1 i}=\left[\Delta t_{m^{\prime \prime} / C R_{i}}, \Delta t_{m^{\prime} / C R_{i}}\left[, I_{2 i}=\left[\Delta t_{m^{\prime} / C R_{i}}, \Delta t_{m / C R_{i}}\left[, I_{3 i}=\left[\Delta t_{m / C R_{i}}, \Delta t_{M / C R_{i}}\left[, I_{4 i}=\left[\Delta t_{M / C R_{i}}, \Delta t_{M^{\prime} / C R_{i}}[\right.\right.\right.\right.\right.\right.\right.$ and $I_{5 i}=\left[\Delta t_{M^{\prime} / C R_{i}}, \Delta t_{M^{\prime \prime} / C R_{i}}[\right.$.

The margin of passive robustness is available in $\left(I_{2 i} \cup I_{4 i}\right)$ whereas the margin of active robustness is in $\left(I_{1 i} \cup I_{5 i}\right)$. From a functional point of view, there are three intervals of use in which it is possible to prove the validity: interval of normal functioning, interval of passive robustness and interval of active robustness. In the case of an abnormal functioning, there is always duality of advance and delay scenarios.

The adopted filtering mechanism is described by the interpreted T-time Petri net of the figure 4 . Several cases can arise [11].

- If there are absence of order (not $A_{i}$ ) and presence of $C R_{i}$, there are freezing of the control and generation of a symptom $S_{i}^{2}$ (place $p_{3}$ ).

- If the sensor signal $C R_{i}$ arrives in the time interval $\left[0, \Delta t m^{\prime \prime}{ }_{C R_{i}}\right.$, there are freezing of the control and generation of a symptom $S_{i}^{2}$ (place $\left.p_{3}\right)$.

- If the sensor signal $C R_{i}$ arrives in the time interval $I_{1 i}=\left[\Delta t_{m^{\prime \prime}} / C R_{i}, \Delta t_{m^{\prime}} / C R_{i}[\right.$, there are change of the control (active robustness to an advance) and memorizing a symptom $S_{i}^{2}$ (place $p_{4}$ ).

- If the sensor signal $C R_{i}$ arrives in the time interval $I_{2 i}=\left[\Delta t_{m^{\prime}} / C R_{i}, \Delta t_{m / C R_{i}}\right.$, there is no change of the control (passive robustness to an advance) but only a memorizing of a symptom $S_{i}^{2}$ (place $p_{5}$ ).

- If the sensor signal $C R_{i}$ arrives in the time interval $I_{3 i}=\left[\Delta t_{m / C R_{i}}, \Delta t_{M / C R_{i}}\right.$, the behavior of the system is normal (place $p_{6}$ ). 


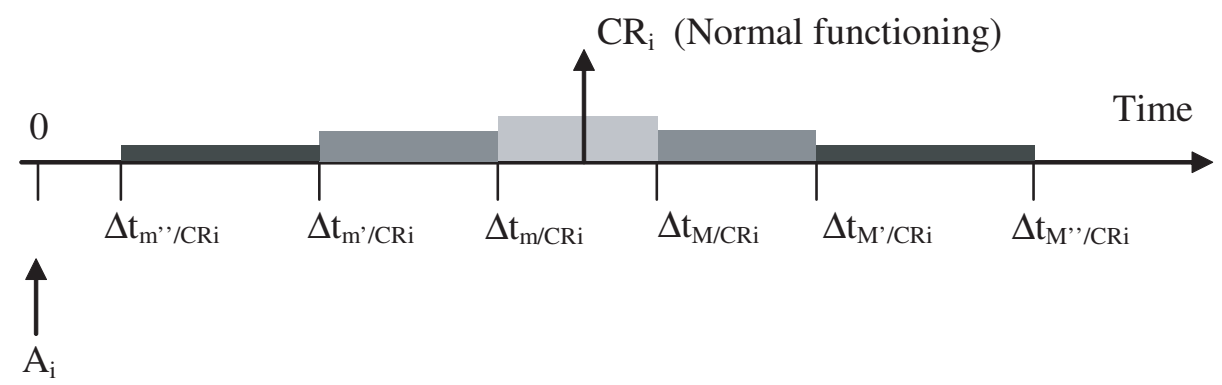

(Start-Event)

Normal functioning interval

Passive robustness interval

- Active robustness interval

Figure 3: Robustness integration in the operation associated model

- At the instant $\Delta t_{M / C R_{i}}\left(\operatorname{transition} t_{7}\right.$ ), there is automatically memorizing of a symptom $S_{i}^{1}$ (place $\left.p_{7}\right)$.

- If the sensor signal $C R_{i}$ arrives in the time interval $I_{4 i}=\left[\Delta t_{M / C R_{i}}, \Delta t_{M^{\prime} / C R_{i}}\right.$, it is a case of passive robustness to a delay (place $p_{8}$ ). The symptom $S_{i}^{1}$ is already memorized (place $p_{7}$ ).

- If the sensor signal $C R_{i}$ arrives in the time interval $I_{5 i}=\left[\Delta t_{M^{\prime} / C R_{i}}, \Delta t_{M^{\prime \prime} / C R_{i}}[\right.$, a change of the control is necessary (active robustness to a delay, place $p_{9}$ ).

- At the instant $\Delta t_{M^{\prime \prime} / C R_{i}}$ (transition $t_{10}$ ), there is freezing of the control (place $p_{10}$ ).

\section{Localization of time disturbances in a given topology}

When, for example, the filtering mechanism executes a control freezing, it is necessary to know where the initial disturbance was occurred. This task is performed on a model of the workshop which uses P-time Petri net in order to integrate the staying time constraints in its structure. This aspect is presented in the following section.

\subsection{Controlled P-time Petri net}

The formal definition of a P-time Petri net is given by a pair $\langle R$; IS $\rangle$, where [12]:

- $R$ is a marked Petri net,

- IS : $P \rightarrow\left(Q^{+} \cup 0\right) \times\left(Q^{+} \cup+\infty\right)$

$p_{i} \rightarrow I S_{i}=\left[a_{i}, b_{i}\right]$ with $0 \leq a_{i} \leq b_{i}$.

$I S_{i}$ defines the static interval of staying time of a mark in the place $p_{i}$ belonging to the set of places $P$ $\left(Q^{+}\right.$is the set of positive rational numbers). A mark in the place $p_{i}$ is taken into account in transition validation when it has stayed in $p_{i}$ at least a duration $a_{i}$ and no longer than $b_{i}$. After the duration $b_{i}$ the token will be dead.

Using [15], controlled P-time Petri net is defined as a quadruplet $R p c=\left(R p, \varphi, U, U_{0}\right)$ such that:

- $R p$ is a P-time Petri net which describes the opened loop system, 
Time Disturbances and Filtering of Sensors Signals

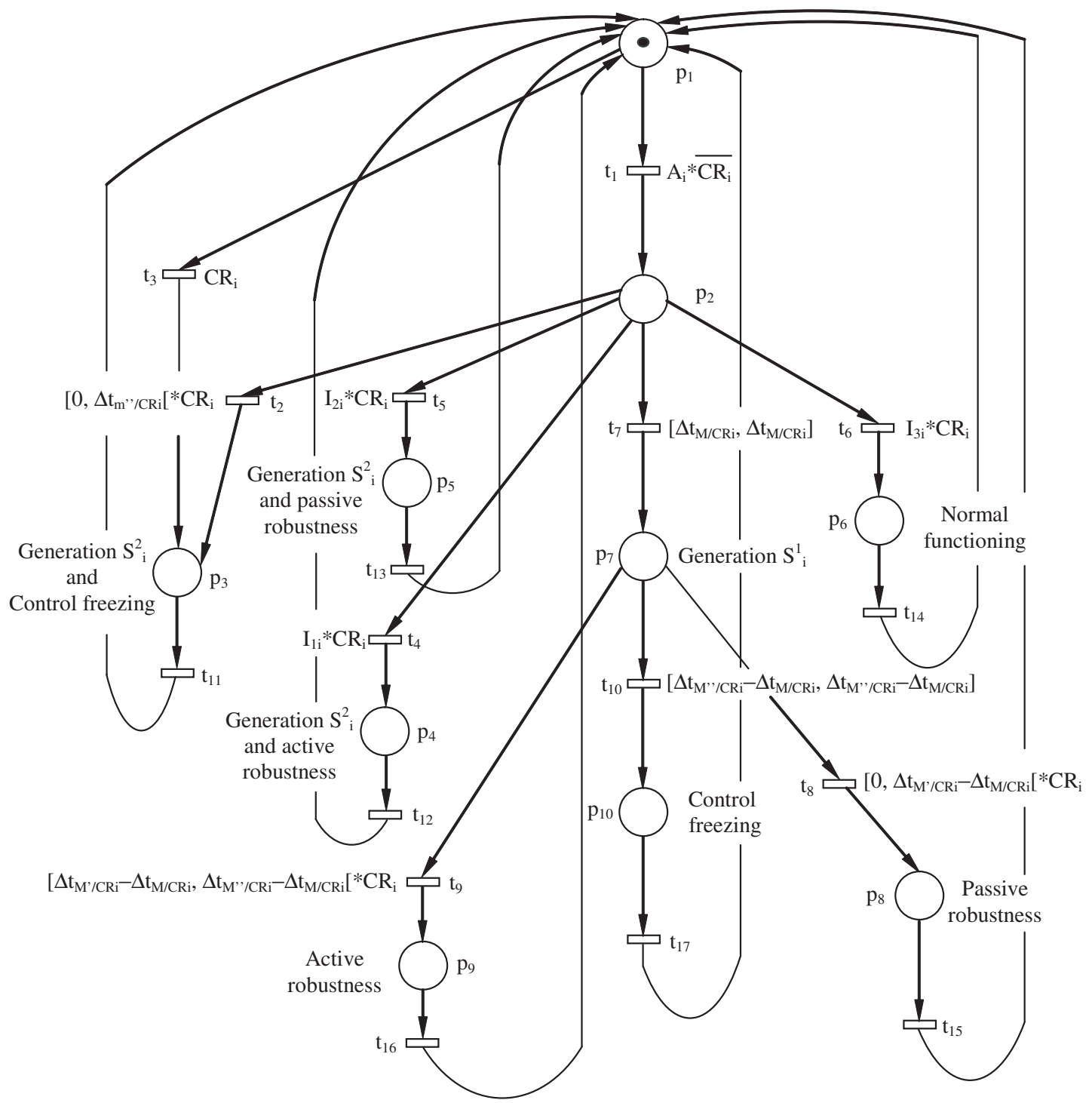

Figure 4: Robustness integration in the filtering mechanism of sensors signals 
- $\varphi$ is an application from the set of places $(P)$ toward the set of operations $(\Gamma): \varphi: P \rightarrow \Gamma$,

- $U$ is the external control of the set of transitions $(T)$ built on the predicates using the occurrence of internal or external observable events of the system: $U: T \rightarrow\{0,1\}$,

- $U_{0}$ is the initial value of the predicate vector.

Let us denote by:

- $T_{O} \quad$ : the set of observable transitions,

- $T_{U O} \quad$ : the set of non observable transitions,

- $T_{S} \quad$ : the set of synchronization transitions,

- $T_{N S} \quad$ : the set of non synchronization transitions,

- $T_{P} \quad$ : the set of parallelism transitions,

- $t_{i}^{\circ}\left(\operatorname{resp} .{ }^{\circ} t_{i}\right):$ the output (resp. the input) places of the transition $t_{i}$,

- $p_{i}^{\circ}\left(\operatorname{resp} .{ }^{\circ} p_{i}\right)$ : the output (resp. the input) transitions of the place $p_{i}$,

- $q_{i e} \quad:$ the expected sojourn time of the token in the place $p_{i}$,

- $S t_{e}(n) \quad$ : the $n^{\text {nd }}$ expected firing instant of the transition $t$,

- St $(n) \quad$ : the $n^{n d}$ effective firing instant of the transition $t$.

\subsection{Functional decomposition}

A workshop in repetitive functioning mode is modeled by a Strongly Connected Event Graph (SCEG) [13]. Performances of a SCEG running in mono-periodic functioning mode are proved to be the same as when using the K-periodic functioning [13]. Consequently, a mono-periodic functioning is used in order to decrease the complexity of the supervisory problem [14]. In this case, for each transition t, $S t_{e}(n+1)=S t_{e}(n)+\pi_{0}$ where $\pi_{0}$ is the period of the periodic functioning of the given discrete event system. In this paper, the scheduling task is supposed to be done. Therefore, the SCEG corresponding to the system is provided. Moreover, the setting of transitions firing instants is fixed too.

As the sojourn times in places have not the same functional signification when they are included in the sequential process of a product or when they are associated to a free resource, a decomposition of the P-time Petri net model into four sets is made using [15]. The assumption of multi-product job-shops without assembling tasks as it was established in [16] is used:

- $R_{U}$ is the set of places representing the used machines,

- $R_{N}$ corresponds to the set of places representing the free machines which are shared between manufacturing circuits,

- $\operatorname{Trans}_{C}$ is the set of places representing the loaded transport resources,

- $\operatorname{Trans}_{N C}$ is the set of places representing the unloaded transport resources (or the interconnected buffers). 
Figure 5, shows a P-time Petri net $(G)$ modeling a system composed by two sequential processes $G O_{1}$ and $\mathrm{GO}_{2}$ with two shared machines $\left(M_{1}, M_{2}\right)$, where:

$R_{U}=\left\{p_{2}, p_{4}, p_{11}, p_{13}, p_{15}\right\}, R_{N}=\left\{p_{6}, p_{7}, p_{8}, p_{9}\right\}, \operatorname{Trans}_{C}=\left\{p_{1}, p_{3}, p_{10}, p_{12}, p_{14}\right\}$, Trans $_{N C}=\left\{p_{5}, p_{16}\right\}, G O_{1}=\left(t_{12}, p_{10}, t_{6}, p_{11}, t_{7}, p_{12}, t_{8}, p_{13}, t_{9}, p_{14}, t_{10}, p_{15}, t_{11}\right)$ and $G O_{2}=$ $\left(t_{5}, p_{1}, t_{1}, p_{2}, t_{2}, p_{3}\right.$,

$\left.t_{3}, p_{4}, t_{4}\right)$.

The intervals $\left(I S_{i}\right)$ and the expected staying times $\left(q_{i e}\right)$ associated to the places $\left(p_{i}\right)$ are:

$I S_{1}=[30,50], q_{1 e}=38, I S_{2}=[5,12], q_{2 e}=7, I S_{3}=[10,20], q_{3 e}=15, I S_{4}=[5,20], q_{4 e}=10$, $I S_{5}=[1,+\infty], q_{5 e}=10, I S_{6}=[0,+\infty], q_{6 e}=5, I S_{7}=[0,+\infty], q_{7 e}=8, I S_{8}=[8,+\infty], q_{8 e}=13$, $I S_{9}=[8,+\infty], q_{9 e}=15, I S_{10}=[5,15], q_{10 e}=12, I S_{11}=[15,20], q_{11 e}=17, I S_{12}=[3,7], q_{12 e}=6$, $I S_{13}=[2,20], q_{13 e}=5, I S_{14}=[2,7], q_{14 e}=5, I S_{15}=[15,20], q_{15 e}=16, I S_{16}=[1,+\infty]$ and $q_{16 e}=19$. The initial expected firing instants of each transition are:

$S t_{1 e}(1)=15, S t_{2 e}(1)=22, S t_{3 e}(1)=37, S t_{4 e}(1)=7, S t_{5 e}(1)=17, S t_{6 e}(1)=12, S_{7 e}(1)=29, S_{8 e}(1)=$ $35, S t_{9 e}(1)=0, S t_{10 e}(1)=5, S t_{11 e}(1)=21$ and $S t_{12 e}(1)=0$.

The repetitive functioning mode is characterized by the period $\pi_{0}=40$.

Definition 3. A mono-synchronized subpath is a path containing one and only one synchronization transition which is its last node.

Definition 4. An elementary mono-synchronized subpath is a mono-synchronized subpath beginning with a place $p$ such as ${ }^{\circ} p$ is a synchronization transition.

In figure 5, there are eight elementary mono-synchronized subpaths constituting a partition of $G$ : $L p_{1}=\left(p_{13}, t_{9}, p_{14}, t_{10}, p_{15}, t_{11}, p_{16}, t_{12}, p_{10}, t_{6}\right), L p_{2}=\left(p_{13}, t_{9}, p_{9}, t_{1}\right), L p_{3}=\left(p_{2}, t_{2}, p_{3}, t_{3}\right)$, $L p_{4}=\left(p_{2}, t_{2}, p_{8}, t_{8}\right), L p_{5}=\left(p_{4}, t_{4}, p_{5}, t_{5}, p_{1}, t_{1}\right), L p_{6}=\left(p_{4}, t_{4}, p_{6}, t_{6}\right), L p_{7}=\left(p_{11}, t_{7}, p_{7}, t_{3}\right)$ and $L p_{8}=\left(p_{11}, t_{7}, p_{12}, t_{8}\right)$.

Property 1. A place $p_{m p}$ belonging to a sequential process represents a shared machine if and only if $p_{m p}^{\circ} \in T_{P}$ or ${ }^{\circ} p_{m p} \in T_{S}$.

Property 2. The first node of an elementary mono-synchronized subpath is a place belonging to $R_{U}$ and representing a shared machine.

\subsection{Time disturbances localization}

Let us remember some definitions.

Definition 5. A time disturbance is detectable if, when it occurs, there exists at least one transition $t \in T_{O}$ such as $S t(n) \neq S t_{e}(n)$.

Definition 6. A time disturbance is quantifiable if its value can be analytically known.

Definition 7. A time disturbance is localizable when its occurrence node can be identified.

Definition 8. A time disturbance is partially localizable when its occurrence node location can be proved to belong to a given subset of $P$.

Definition 9. A time disturbance is observable when it is detectable, quantifiable and localizable.

Definition 10. The time passive rejection capacity interval of a path $L p$ is $R C(L p)=[C a(L p), C r(L p)]$ where:

$$
C a(L p)=\sum_{p_{i} \in\left(L p \cap\left(R_{N} \cup \operatorname{Trans}_{N C}\right)\right)}\left(q_{i e}-b_{i}\right),
$$




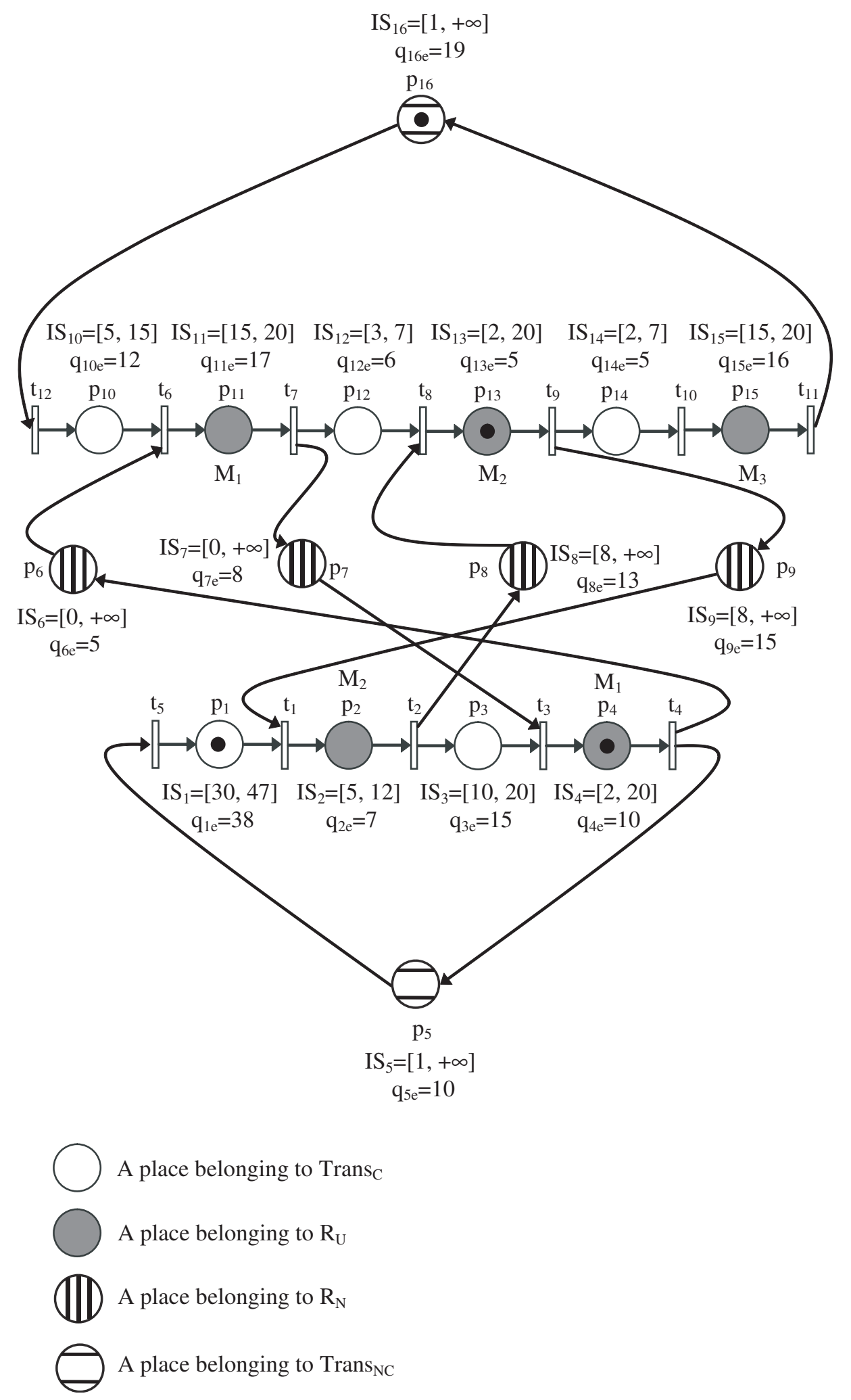

Figure 5: An Hillion like model with functional decomposition 


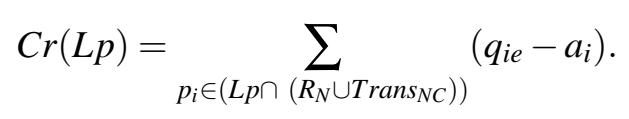

$\mathrm{Ca}(\mathrm{Lp})$ (resp. $\mathrm{Cr}(\mathrm{Lp}))$ is called the time passive rejection capacity for an advance (resp. a delay) time disturbance occurrence.

Definition 11. Let $\delta$ a time disturbance and $\mathrm{SN}$ a set of nodes belonging to a P-time Petri net. $\delta \in S N$ (resp. $\delta \notin S N$ ) means that the occurrence of $\delta$ is (resp. is not) in a node of SN.

\section{Used notations:}

- $C_{s e}$ is the set of elementary mono-synchronized subpaths.

- $I N(L p)$ is the first node of the path $L p$.

- $O U T(L p)$ is the last node of the path $L p$.

- $L p\left(t^{*}, t\right)$ is the oriented subpath of $L p$ beginning with $t^{*}$ and ending with $t$.

- $M_{n-1}\left(L p\left(t^{*}, t\right)\right)$ is the number of tokens in $L p\left(t^{*}, t\right)$ after the completion of the cycle $(n-1)$.

- Given a time disturbance $\delta, \delta r_{t}(n)$ is the resulting residue quantified at the transition $t$ which is fired at $S t(n)$.

- $E C\left(I N^{\circ}(L p), t\right)$ is the set of oriented paths connecting the node $I N^{\circ}(L p)$ of the path $L p$ to the transition $t$.

- $H\left(I N^{\circ}(L p), t\right)=\min _{L_{i} \in\left[E C\left(I N^{\circ}(L p), t\right) \backslash L p\left(I N^{\circ}(L p), t\right)\right]}\left(C r\left(L_{i}\right)\right)+\delta r_{t}(n)$.

- $H^{\prime}\left(I N^{\circ}(L p), t\right)=\min _{L_{i} \in E C\left(I N^{\circ}(L p), t\right)}\left(C r\left(L_{i}\right)\right)+\delta r_{t}(n)$.

Lemma 12. Let $L p \in C_{s e}, t \in\left(L p \cap T_{O} \cap T_{N S}\right), t^{*} \in\left(L p \cap T_{O}\right)$ and $\delta$ a time disturbance having a residue $\delta r_{t}(n) \neq 0$ quantified at the transition $t$. The following results are established [17]:

$$
\begin{aligned}
& \delta r_{t *}\left(n-M_{n-1}\left(L p\left(t^{*}, t\right)\right)\right)=0 \Longrightarrow \delta \in\left[L p\left(t^{*}, t\right) \backslash\left\{t^{*}\right\}\right], \\
& \delta r_{t *}\left(n-M_{n-1}\left(L p\left(t^{*}, t\right)\right)\right) \neq 0 \Longrightarrow \delta \notin\left[L p\left(t^{*}, t\right) \backslash\left\{t^{*}\right\}\right] .
\end{aligned}
$$

This lemma discusses the case of two observable transitions, $t$ and $t^{*}$, such that $t$ is not a synchronization one. When a disturbance is detected at a downstream transition $t$ and is not detected at $t^{*}$, it is generated between these two transitions. Otherwise, the disturbance occurrence is outside the restriction of the considered path that connects $t^{*}$ to $t$.

Lemma 13. Let $L p \in C_{s e}, t \in\left(L p \cap T_{O}\right), t p \in\left(L p \cap T_{P}\right), I_{L p}=\left\{L_{i} \in C_{s e} / O U T\left(L_{i}\right)={ }^{\circ} I N(L p)\right\}$ and $\delta$ a time disturbance having a residue $\delta r_{t}(n)>0$ quantified at the transition $t$. The following assertion is true [17]:

$$
\begin{gathered}
\delta r_{t p}\left(n-M_{n-1}(L p(t p, t))\right)<H^{\prime}(t p, t) \Longrightarrow \\
\delta \notin\left\{\bigcup_{L_{i} \in I_{L p}}\left\{L_{i} \backslash\left\{I N\left(L_{i}\right), I N^{\circ}\left(L_{i}\right)\right\}\right\} \bigcup\left\{{ }^{\circ} t p, t p\right\}\right\} .
\end{gathered}
$$


In other words, when the residue of the disturbance at the parallelism transition $t p$ does not justify the residue at the transition $t$, forcibly the disturbance has not crossed $t p$.

Lemma 14. Let $L p \in C_{s e}, t \in\left(L p \cap T_{O} \cap T_{S}\right), t^{*} \in\left(L p \cap T_{O}\right)$ and $\delta$ a time disturbance having a residue $\delta r_{t}(n)>0$ quantified at the transition $t$. The following results are established [17]:

$$
\begin{gathered}
\delta r_{t *}\left(n-M_{n-1}\left(L p\left(t^{*}, t\right)\right)\right)=0 \Longrightarrow \delta \notin\left[L p\left(I N^{\circ}(L p), t^{*}\right) \backslash\left\{I N^{\circ}(L p)\right\}\right], \\
\left\{\begin{array}{l}
0 \leq C r\left(L p\left(I N^{\circ}(L p), t^{*}\right)\right)<H\left(I N^{\circ}(L p), t\right) \\
\delta r_{t *}\left(n-M_{n-1}\left(L p\left(t^{*}, t\right)\right)\right)=0
\end{array}\right. \\
\left\{\begin{array}{l}
\delta \notin\left[\left(L p \backslash L p\left(t^{*}, t\right)\right) \cup\left\{t^{*}\right\}\right] \\
\delta r_{I N^{\circ}(L p)}\left(n-M_{n-1}\left(L p\left(I N^{\circ}(L p), t\right)\right)\right)<H\left(I N^{\circ}(L p), t\right)
\end{array}\right. \\
\left\{\begin{array}{l}
\delta r_{t *}\left(n-M_{n-1}\left(L p\left(t^{*}, t\right)\right)\right) \neq 0 \\
\delta r_{t}(n)+C r\left(L p\left(t^{*}, t\right)\right) \neq \delta r_{t *}\left(n-M_{n-1}\left(L p\left(t^{*}, t\right)\right)\right)
\end{array}\right. \\
\delta \notin\left[L p\left(I N^{\circ}(L p), t\right) \backslash\left\{I N^{\circ}(L p)\right\}\right] .
\end{gathered}
$$

The above lemma discusses the case of two observable transitions, $t$ and $t^{*}$, such that $t$ is a synchronization one. Several results are given.

If the residue at the transition $t^{*}$ is equal to zero, the disturbance does not belong to the restriction of $L p$ between its only parallelism transition $I N^{\circ}(L p)$ and $t^{*}$.

If the disturbance has crossed the parallelism transition of $L p\left(I N^{\circ}(L p)\right)$ and if its residue at $I N^{\circ}(L p)$ is greater than the passive rejection capacity of the restriction of $L p$ between $I N^{\circ}(L p)$ and $t^{*}$, the residue at $t^{*}$ must be different of zero. Otherwise, the disturbance has not crossed $I N^{\circ}(L p)$.

If the residue at $t^{*}$ is different of zero and if it does not justify the residue at the transition $t$, the occurrence of the disturbance is not in the restriction of $L p$ between $I N^{\circ}(L p)$ and $t$.

Lemma 15. Let $L p \in C_{s e}, t p \in\left(L p \cap T_{P} \cap T_{U O}\right), t \in\left(L p \cap T_{O}\right)$ and $C r(L p(t p, t))$ the time passive rejection capacity of $L p$ between tp and t for delay occurrence. Let us call DIF $(t p)$ the set of paths beginning with tp. Let us denote $D I F_{n}(t p)$ the restriction of DIF $(t p)$ such that: $\forall L p^{\prime} \in D I F_{n}(t p), \forall t^{\prime} \in L p^{\prime}$, we have $S t^{\prime}\left(n+m_{t^{\prime}}\right)<\operatorname{St}(n)$ where $m_{t^{\prime}}=M_{n-1}\left(L p^{\prime}\left(t p, t^{\prime}\right)\right)-M_{n-1}(L p(t p, t))$.

Now, let $L p^{\prime} \in D I F_{n}(t p), t^{*} \in\left(L p^{\prime} \cap T_{O}\right)$ and $C r\left(L p^{\prime}\left(t p, t^{*}\right)\right)$ the passive rejection capacity of $L p^{\prime}$ between t $p$ and $t^{*}$. Given a delay time disturbance $\delta$, the following results are true [17]:

$$
\begin{gathered}
\left\{\begin{array}{l}
\left(t \notin T_{S}\right) \wedge\left(\delta r_{t}(n)>0\right) \\
\delta r_{t}(n)+C r(L p(t p, t))-C r\left(L p^{\prime}\left(t p, t^{*}\right)\right)>0 \Longrightarrow \delta \in[L p(t p, t) \backslash\{t p\}], \\
\delta r_{t *}\left(n+m_{t *}\right)=0
\end{array}\right. \\
\left\{\begin{array}{l}
\left(t \notin T_{S}\right) \wedge\left(\delta r_{t}(n)>0\right) \\
\delta r_{t *}\left(n+m_{t *}\right) \neq 0
\end{array}\right. \\
\left\{\begin{array}{l}
\left(t \in T_{S}\right) \wedge\left(\delta r_{t}(n)>0\right) \\
C r\left(L p^{\prime}(t p, t)\right)<H^{\prime}(t p, t) \\
\delta r_{t *}\left(n+m_{t *}\right)=0
\end{array}\right. \\
\left\{\begin{array}{l}
\delta \notin\left\{{ }^{\circ} t p, t p\right\} \\
\delta r_{t p}\left(n-M_{n-1}(L p(t p, t))\right)<H^{\prime}(t p, t)
\end{array}\right) \\
\left\{\begin{array}{l}
\left(t \in T_{S}\right) \wedge\left(\delta r_{t}(n)>0\right) \\
\delta r_{t *}\left(n+m_{t *}\right) \neq 0
\end{array}\right.
\end{gathered}
$$


When $t p$ is a non observable parallelism transition, the following assertion may be used: if a disturbance modifies the $t p$ firing instant, it must be seen downstream of $t p$. Consequently, when the value of the residual effect of the disturbance is greater than the rejection capacity of a given path, a residual variation has to be observed.

The different lemmas formulated constitute a tool aiming to define the set of nodes where the disturbance may occur and the subset where it is proved that it did not occur. Then the question of using the above lemmas in order to make them collaborate has to be tackled. In other words, it remains to establish an algorithm using these lemmas while testing all mono-synchronized subpaths of the given P-time Petri net model.

\section{Conclusions}

This paper deals with supervision in critical time manufacturing job-shops. In such systems operation times are included between a minimum and a maximum value. A filtering mechanism of sensors signals integrating the robustness values is described. It provides the avoidance of control freezing if the time disturbance is in the robustness intervals. Therefore, it makes it possible to continue the production in a degraded mode providing the guarantees of quality and safety. It should be noted that the knowledge of robustness intervals is a significant parameter in the proposed mechanism. The assumptions formulated in these lines are very restrictive. It is natural to consider different scenarios where the temporal specifications of the process are not fulfilled, nevertheless the production can continue. It is necessary to introduce a finer classification of abnormal functioning and their impact on the considered systems. In this context, fuzzy logic can be used.

When a symptom of an abnormal functioning is claimed by the filtering mechanism, it is imperative to localize the time disturbance occurrence. Based upon controlled P-time Petri nets as a modeling tool, a series of lemmas are quoted in order to build a theory dealing with localization problem. This is quite useful for the maintenance task.

In the near future, it is essential to develop an algorithm using the lemmas results and providing localization of time disturbances.

\section{References}

[1] S. Calvez, P. Aygalinc, and P. Bonhomme, Proactive/Reactive Approach for Maintenance Tasks in Time Critical Systems, IEEE International Conference on Emerging Technologies and Factory Automation (ETFA'2005), Catane, Vol. 1, pp. 947-953, September 2005.

[2] S. Collart Dutilleul, and E. Craye, Performance and tolerance evaluation, SAFEPROCESS'03, IFAC Symp. on Fault Detection, Supervision and Safety for Technical Processes, Washington, June 2003.

[3] A. Boufaied, A. Subias, and M. Combacau, Chronicle modeling by Petri nets for distributed detection of process failures, IEEE Conference on Systems, Man, and Cybernetics (SMC'02), Hammamet, October 2002.

[4] P. Declerck, and M. K. Didi Alaoui, Modelling and analysis of P-time event graphs in the (min, max, +) algebra, IEEE Conference on Systems, Man, and Cybernetics (SMC'04), The Hague, Vol. 2, pp. 1807-1812, October 2004.

[5] N. Jerbi, S. Collart Dutilleul, E. Craye, and M. Benrejeb, Observability of Tolerant Multi-product Job-shops in Repetitive Functioning Mode, IMACS'05, Paris, July 2005. 
[6] A. E. K. Sahraoui, Contribution à la surveillance et à la commande d'ateliers, Ph.D. Thesis, Université Paul Sabatier, Toulouse, 1987.

[7] A. Toguyeni, E. Craye, and J. C. Gentina, A method of temporal analysis to perform on-line diagnosis in the context of Flexible Manufacturing System, IECON'90, Vol. 1, pp. 445-450, Pacific GroveCalifornia, November 1990.

[8] M. Nourelfath, Extension de la théorie de la supervision à la commande des systèmes à événements discrets: application à la sécurité opérationnelle des systèmes de production, Ph.D. Thesis, INSA de Lyon, France, July 1997.

[9] A. Toguyeni, Surveillance et diagnostic en ligne dans les ateliers flexibles de l'industrie manufacturière, Ph.D. Thesis, Université des Sciences et Technologies de Lille, November 1992.

[10] M. Diaz, Les réseaux de Petri - Modèles fondamentaux, Ed. Hermès, Paris, 2001.

[11] N. Jerbi, S. Collart Dutilleul, E. Craye, and M. Benrejeb, Intégrat0on de la robustesse dans la supervision de systèmes manufacturiers à contraintes de temps, Conférence Internationale Francophone d'Automatique (CIFA'06), Bordeaux, May 2006.

[12] W. Khansa, P. Aygalinc, and J. P. Denat, Structural analysis of P-Time Petri Nets, Computational Engineering in Systems Applications (CESA'96), Lille, pp. 127-136, July 1996.

[13] S. Laftit, J. M. Proth, and X. Xie, Optimisation of invariant Criteria for Event Graph, IEEE Trans. on Automatic Control, Vol. 37, no. 5, pp. 547-555, May 1992.

[14] S. Collart Dutilleul, J. P. Denat, and W. Khansa, Use of Periodic Controlled Petri Net for Discrete Event Dynamical System Control Synthesis, ECC'95, Rome, pp. 2060-2065, September 1995.

[15] J. Long, and B. Descotes-Genon, Flow Optimization Method for Control Synthesis of Fexible Manufacturing Systems Modeled by Controlled Timed Petri Nets, IEEE International Conference on Robotics and Automation, Atlanta, Georgia, Vol. 1, pp. 598-603, May 1993.

[16] H. P. Hillion, and J. M. Proth, Performance evaluation of job-shop systems using timed event graphs, IEEE Trans. on Automatic Control, Vol. 34, no. 1, pp. 3-9, 1989.

[17] N. Jerbi, S. Collart Dutilleul, E. Craye, and M. Benrejeb, Localization of Time Disturbances in Tolerant Multiproduct Job-shops Without Assembling Tasks, Computational Engineering in Systems Applications (CESA'06), Beijing, pp. 45-50, October 2006.

Nabil Jerbi ${ }^{1,2}$, Simon Collart Dutilleul ${ }^{1}$, Etienne Craye ${ }^{1}$, Mohamed Benrejeb ${ }^{2}$

${ }^{1}$ Ecole Centrale de Lille Laboratoire d'Automatique, Génie Informatique et Signal Cité Scientifique, BP 48, 59651 Villeneuve d'Ascq, France

${ }^{2}$ Ecole Nationale d'Ingénieurs de Tunis Unité de recherche LARA-Automatique BP 37, Le Belvédère, 1002 Tunis, Tunisie

E-mail: nabil.jerbi@isetso.rnu.tn, simon.collart_dutilleul@ec-lille.fr, etienne.craye@ec-lille.fr, mohamed.benrejeb@enit.rnu.tn 